\title{
From Molecules to Patients: The Clinical Applications of Translational Bioinformatics
}

\author{
K. Regan, P. R.O. Payne \\ The Ohio State University, Department of Biomedical Informatics, Columbus, OH, USA
}

\begin{abstract}
Summary
Objective: In order to realize the promise of personalized medicine, Translational Bioinformatics (TBI) research will need to continue to address implementation issues across the clinical spectrum. In this review, we aim to evaluate the expanding field of TBI towards clinical applications, and define common themes and current gaps in order to motivate future research. Methods: Here we present the state-of-the-art of clinical implementation of TBI-based tools and resources. Our thematic analyses of a targeted literature search of recent TBl-related articles ranged across topics in genomics, data management, hypothesis generation, molecular epidemiology, diagnostics, therapeutics and personalized medicine.

Results: Open areas of clinically-relevant TBI research identified in this review include developing data standards and best practices, publidy available resources, integrative systemslevel approaches, user-friendly tools for dinical support, cloud computing solutions, emerging technologies and means to address pressing legal, ethical and social issues. Conclusions: There is a need for further research bridging the gap from foundational TBI-based theories and methodologies to clinical implementation. We have organized the topic themes presented in this review into four conceptual foci - domain analyses, knowledge engineering, computational architectures and computation methods alongside three stages of knowledge development in order to orient future TBl efforts to accelerate the goals of personalized medicine.
\end{abstract}

\section{Keywords}

Translational bioinformatics, clinical informatics, personalized medicine, clinical research, translational science

Yearb Med Inform 2015;10:164-9 http://dx.doi.org/10.15265/IY-2015-005

Published online August 13, 2015

\section{Introduction}

The field of Translational Bioinformatics

(TBI) can be defined as being concerned with: "The development of storage, analytic, and interpretive methods to optimize the transformation of increasingly voluminous biomedical data into proactive, predictive, preventative, and participatory health."[1]. In this capacity, TBI research and development efforts seek to enable the efficient and timely translation of bio-molecular discoveries into actionable knowledge relevant to clinical end-points, so as to advance the state of human health and wellness. In doing so, a driving framework for TBI, per the aforementioned definition, is that of personalized medicine. The primary objective of personalized medicine is to ensure that patient outcomes are optimized through the delivery of preventative measures and/or treatments that are customized to meet patient-level needs and characteristics and that are informed by the best possible science. Weston and Hood can be credited with using the label "P4 Medicine" to first describe this paradigm-shifting approach to healthcare. They posited that fundamental approaches to health promotion, disease diagnosis, and treatment planning, must shift from being reactive to proactive, wherein such health promotion and care delivery efforts become predictive, personalized, preventive and participatory [2]. Such models are incredibly information intensive, requiring the systematic application of Biomedical Informatics (BMI) theories and methods in order to discover and manage large volumes of heterogeneous data, apply analytical techniques to develop insights from such data resources, and ultimately, deliver ensuing knowledge in an actionable format at any number of clinically relevant end-points. In response to this need, there is a growing and robust body of reports in the peer-reviewed literature that describe the design and verification or validation of TBI tools and methods that can achieve such translation and knowledge generation [3, 4]. However, many if not all of these reports tend to focus on foundational methodology development. In comparison, the number of reports concerning the implementation science issues that exist when seeking to apply novel TBI knowledge at the individual patient or population levels remains relatively modest. In response to this gap in knowledge and practice, we examined the current state-of-the-art in terms such clinical implementation of TBI-generated knowledge. Here we present a set of common thematic elements that can frame open research questions that may serve to catalyze future directions in the TBI field. In doing so, it is our intent to motivate continued research and development targeting such translational junctures so as to demonstrate the value of TBI in advancing the clinical and life sciences.

\section{Methods}

In order to evaluate the current state-of-theart and future directions for implementation level work at the intersection of TBI and one or more clinical end-points, we conducted a targeted assessment of a convenience sample of peer-reviewed literature using methods summarized below:

1) Using the PubMed interface to the Medline database, we searched for literature published between January 1, 2012 and December 1, 2014 in which the term "Translational Bioinformatics" or "TBI" appeared in 
the title or abstract of a given report (using the specific query formulation as follows: "( ( ( "Translational Bioinformatics" [Title/ Abstract]) OR "TBI"[Title/ Abstract]) AND ("2012/01/01" [Date - Publication]: "2014/12/31" [Date Publication])) ) AND "Informatics" [MeSH Terms]"). This yielded an initial corpus of 29 articles. Of note, we utilized the acronym "TBI" due to its increasing use in publications in the field, recognizing the ambiguity with other uses of the same acronym, thus necessitating the manual filtering/censoring step described below as part of the overall literature search process.

2) An additional inclusion criterion for identified manuscripts was then employed, requiring that such publication appeared in peer-reviewed biomedical or health related journal. This was achieved by using the filters available in the PubMed interface that restrict search results to those publications classified as either "Core Clinical Journals" or "MEDLINE". This reduced the corpora to 22 articles.

3) Subsequently, a heuristic review of the abstracts and introductions was performed by a single subject matter expert (PP) in order to: 1) eliminate articles that describe traumatic brain injury research, which represents an ambiguous use of the aforementioned "TBI" acronym"; and 2) further select for articles that described one or more clinical end-points for their work (e.g., censoring theory/methods papers that do not have clinical foci as part of their design or application). This further reduced the corpora to 18 articles.

4) Further expert review of the literature was performed via the inclusion of additional selected reports identified in the annual AMIA Joint Summits on Translational Science "TBI Year in Review" (http:// rbaltman.wordpress.com). These steps yielded a final corpus of 24 manuscripts focusing on TBI with clinical end-points.

5) Finally, the selected manuscripts were reviewed in detail and subjected to thematic analyses by the authors, as is reported in the remainder of this article. No further inclusion/exclusion criteria were applied.

\section{Review of Selected Literature}

\section{Genomics}

The understanding of the functional impact of genetic variation is a central challenge in realizing the goals of personal genomics. As single nucleotide variants (SNVs) represent the most common type of genetic variation, there is great interest in elucidating the functional effect of SNVs among biologists and clinicians. Capriotti et al describe databases and bioinformatics tools necessary to advance our interpretation of human genetic variation, with particular emphasis SNVs [5]. The authors organize web-based tools for the interpretations of SNVs in 3 categories: predictions of stability change upon mutation, deleterious non-synonymous SNVs and the impact of SNVs at the DNA level. However, there are significant unmet needs prohibiting their use in clinical settings: (i) defining standard, unified protocols for testing functional variation, (ii) designing integrated, publicly available resources of annotated genetic variants, (iii) developing holistic approaches to score the effect of multiple genetic variants and (iv) implementing user-friendly tools for the health care context. These will need to be met in order to generate accurate disease-specific protocols for estimating the risks of disease development and transmission due to personal genome variation. In bridging the translational "T1" gap from basic research discoveries regarding personal genome variants to clinical studies, Simon and Roychowdhury discuss challenges in incorporating cancer genome data in clinical trial design, including the need to define the molecular taxonomy of cancer subtypes, align available drugs to actionable molecular aberrations and institute rapid turnaround of clinical grade sequencing of enrolled patients [6]. The incorporation of next-generation sequencing in clinical trials has the potential to accelerate the development of companion diagnostics for approved drugs, which can relay important information on the potential safety and efficacy of a corresponding therapeutic agent.

\section{Data Management}

Modern biomedical informatics theory and practice has demonstrated the distinct benefits associated with the use of knowl- edge-based systems for the integration of large scale, multi-dimensional clinical phenotype and biomolecular datasets. We recently proposed a conceptual model for knowledge integration in the translational sciences using a knowledge-based system, which is defined as an intelligent agent that employs a computationally tractable knowledge base or repository in order to reason upon data in a targeted domain and reproduce expert performance relative to such reasoning operations [7]. Examples of the application of knowledge-based systems in biomedicine span a broad spectrum, from clinical decision support, to epidemiologic surveillance of public data, to the discovery of novel hypotheses in large-scale research datasets. We introduce the knowledge engineering cycle and a practical model for the design and execution of translational informatics projects in order to promote awareness of required inputs and anticipated outputs, as well as the interrelationships between and across phases. Research in the basic sciences, clinical and public health domains are typically siloed. Furthermore, integration across these domains are limited to due issues concerning data transfer, access control, and model building. The Electronic Medical Records and Genomics (eMERGE) consortium, a system of academic medical centers that cooperatively share biobanking and genotyping data and capabilities to execute scalable "high-throughput phenotyping" of patient cohorts using electronic medical records. The continued success of eMERGE relies on its ability to institute data normalization and harmonization in both clinical and genomic as prerequisites to valid data inference across aggregated data sources [8]. Cloud computing has also been proposed as a solution to address some of these limitations in order to promote translational data integration and analysis [9]. Cloud computing provides access to larger computer processing power and storage, and can permit the sharing of data in real-time collaboration with other users. Examples of TBI cloud research include: cloud-based implementation for sequence analysis (CloudBLAST), large-scale medical imaging (MapReduce), and EHR sharing and integration systems (HealthVault). 


\section{Hypothesis Generation}

TBI-based resources and tools have enabled the formulation of novel hypotheses leading to important discoveries spanning different levels of translational science. Enrichment analyses have gained popularity for analyzing high-throughput biological data due to its simple implementation and easily interpretable results. Shah et al propose that such analyses may be used as an exploratory tool to generate hypotheses for clinical research via extending these methodologies to disease ontologies [10]. Evidence suggests that the majority of complex diseases are caused by complex genetic and gene-environment interactions, and that network analysis approaches offer sophisticated means for multi-dimensional -omics data integration [11]. Bebek et al highlight translational network-based applications: identifying functional sub-networks in complex disease phenotypes; prioritizing candidate disease genes in genome-wide association studies (GWAS), including "rescuing" associations that appear insignificant under multiple hypothesis testing corrections; and exploiting biochemical networks for identifying synthetic lethal drug combinations and off-target drug effects [12]. Protein-protein interaction (PPI) networks can reveal novel pathways and molecular underpinnings of disease. Gonzalez and Kann describe several new methods developed to extract sub-networks that can be used to understand different aspects of disease progression, and ultimately may be able to generate new diagnostic tools, prognostic markers and drug targets [11]. In order to gain a deeper understanding of individual diseases, however, the authors mandate that TBI efforts need to move from global network characterizations to disease-specific interactomes. Complex genetic relationships that culminate in disease phenotypes, including polygenic diseases, further illustrate the need for TBI approaches to dissect gene-disease interrelationships. As this genetic paradigm represents an "information retrieval" challenge, Sarkar et al exploited a vector space model in order to bridge gene-disease knowledge inferred across established knowledge bases, and they validated a set of plausible diseases via supporting literature
[13]. Further investigation will be required to assess the robustness of these approaches in accurately modeling clinical phenotypes with large-scale biomolecular data. Finally, visual analytics can facilitate efficient detection of complex patterns. A recent prototypical development, Interactive Circos, enables the interactive exploration of molecular and phenotype information to generate hypotheses regarding underlying biological mechanisms [14].

\section{Molecular Epidemiology}

There is an urgent need to accelerate the pace of translating epidemiological discoveries into tangible population health benefits. The field of cancer epidemiology is particularly well positioned to do so given the unprecedented access to cancer-related data and the active engagement of the NCI Epidemiology and Genomics Research Program (EGRP) in advancing research. Lam et al recently described "drivers" of this translational framework including: i) collaboration and team science across a variety of disciplines; ii) emerging technologies, including molecular -omics platforms and those that interrogate the "exposome" and gene-environment interactions; iii) multilevel analyses and interventions; and (iv) knowledge integration of basic, clinical, and population sciences [15].

\section{Diagnostics}

Candidate diagnostic and prognostic biomarkers are increasingly identified in biomedical research; however, these biomarkers are often irreproducible across experiments and very few are implemented in clinical practice. One reason accounting for this variability in biomarker discovery is the highly heterogeneous nature of complex diseases. For instance, clinical oncology is leveraging the capabilities of -omics technologies in biomarker discovery at multiple molecular levels: genetic, gene expression, microRNA, protein, metabolic, text mining and utilization of biomarker knowledge bases [16]. Chen et al describe advances in prostate cancer biomarkers discovered via high-throughput technologies and the potential use of integrative network-based approaches as well as cloud computing in next-generation sequencing (NGS) data processing [17]. Specifically, NGS may facilitate patient stratification for targeted therapies, as it has the capability to generate multiple types of genomic alterations, including mutations, gene fusions, copy number alterations, and epigenetic changes simultaneously in a single test. As cancer is a complex disease that involves the interaction of many biological components, the future of cancer care will rely on panels of diagnostic and prognostic markers derived from rigorous, systems-based TBI interventions.

\section{Therapeutics}

When applied in drug research, TBI integrates and extracts actionable information from large data sets, with the expectation that new knowledge can be generated at multiple levels across the spectrum of genes, subcellular compartments, intact cells, organs and tissues, signaling pathways, as well as drug response in populations and patient subgroups [18]. TBI methods can be applied to all aspects of drug discovery, from compound screening and lead selection, through clinical trial design and management and postmarketing surveillance, to adverse-event reporting and drug repurposing [19]. A significant impact of TBI research is that important questions regarding the optimal use of drugs can be asked and answered purely using computational approaches.

The goal of drug repurposing is that by renewing failed drugs and/or finding novel indications for approved drugs, new therapies may be delivered to patients faster and with a higher success rate. Computational drug repositioning is defined as the process of designing and validating automated workflows that can generate hypotheses for new indications for a drug candidate [20]. Hurle et al describe the major computational techniques used for generating drug repurposing hypotheses, including transcriptomics, side effect profiles and GWAS-based information. Computational approaches may further accelerate proof-of-concept validation experiments and small-scale clinical studies 
for repurposed drug candidates. The authors assert that data produced during the various stages of the drug discovery process may serve as critical resources for developing repurposing methods, including in vivo screenings (mouse phenomes), EHR data and other clinical observation studies.

Another TBI opportunity is the ability to apply mechanism-based drug safety assessments to complement current observational pharmacovigilance efforts. For instance, TBI methods can render hypotheses to predict which patients will be most at risk for adverse events, inform post-marketing surveillance strategies, and propose mechanistic explanations for unexpected "safety signals" [18]. In a recent study, investigators analyzed patterns of off-label drug usage in clinical notes with the goal of reducing the incidence of adverse events and for improving patient safety [21]. In another example, researchers utilized a literature discovery approach along with the analysis of EHRs in order to predict and evaluate potential mechanisms of novel drug-drug interactions [22]. Furthermore, TBI-based approaches are poised to integrate genomics and drug toxicity information, exemplified by Johnson et al in their development of a cost-effective genomics platform (XChip) developed to measure RNA splicing changes that occur in response to drug exposure [23].

The analysis of networks of interacting biomolecules and drugs represents a logical extension of our understanding of disease, treatments and patient responses. Fundamental concepts to guide the analysis and interpretation of drug-target interaction networks and applications for personalized medicine, drug repositioning and adverse event detection are described in a recent review [24]. At the core of the proposed network approaches is the integrated representation of drug-drug, drug-target, and protein-protein interactions as complex maps of nodes (i.e. drugs or gene products) interconnected via edges (i.e. physical interactions or co-expression associations). Networks are subsequently computationally inferred, visualized, and analyzed using multiples data resources (e.g. molecular profiles, drug label information, etc.) in the context of certain diseases and/or tissues. These integrated, systematic analyses, and resulting prediction models enable the discovery of clinically actionable knowledge for the generation of new compounds, treatment optimization, and decision support.

Cloud computing services represent a promising alternative for computational drug discovery, as current computer facilities are unable to handle the increasing amount of available biomolecular and clinical data. Dalpa et al describe the paradigm by which highly scalable, cloud computing approaches can be employed to facilitate multi-dimensional genomic-phenomic association studies, drug repositioning research, and pharmacovigilance monitoring; notwithstanding, researchers and policy makers must define standards for building cloud computing applications for health data that address pressing legal, ethical and social issues before this research can foster innovation in personalized medicine [25].

\section{Personalized Medicine}

The fundamental premise of personalized and precision medicine is that by pairing established clinical-pathological notions of disease with state of the art molecular profiling, improved diagnostic, prognostic and therapeutic strategies may be precisely tailored to patient-specific needs. In order for healthcare providers and patients to fully realize the benefits of precision medicine, Mirnezami et al argue for the establishment of frameworks for streamlining burdensome regulatory processes, re-classification of disease states that incorporate patient-specific biomolecular knowledge, and creation of decision support tools and training programs to aid the physician workforce in the clinical interpretation of personal genomes [26]. The NIH Clinical Sequencing Exploratory Research (CSER) program aims to guide best practices for utilizing sequencing information in clinical care. In a recent study comparing the state of the art for integrating whole-genome and whole-exome sequencing results into the EHR, Tarczy-Hornoch et al discovered several approaches to annotation tools, workflow and report generation [27]. The authors advocate for clinical use of sequencing information via: (i) increased cross-site collaboration in creation, curation, and integration of variant databases/knowledge bases (VDBKBs); (ii) generation of both human-readable and computable reports; (iii) development of standards for automating the translation of information in VDBKBs into active decision support rules; (iv) development of best practices for integrating biomedical informatics into clinical and communication workflows; and (v) collaboration with vendors on adapting their active decision support. An additional unmet need for implementing personalized medicine biomarker discovery is synergizing efforts in organizing tissue banks of high quality samples, standardizing EHR documentation and bioinformatics analyses of multi-dimensional clinical and biomolecular phenotypes [28].

\section{Discussion}

The selected corpus of literature have contributed to the current state of clinically-relevant TBI knowledge and practice, and have exhibited a number of thematic trends, as is illustrated in Figure 1 and described below: - The current work being performed at the intersection of TBI and clinical endpoints can be broadly organized into four conceptual foci: 1) domain analyses, concerned with review of the current state of TBI knowledge and practice and the elucidation of critical gaps and/or future directions; 2) the design or application of knowledge engineering methodologies capable of encoding and delivering TBI derived knowledge in computationally and/or human actionable formats; 3 ) the design and delivery of computational architectures capable of collecting, storing, analyzing, and delivering large-scale and heterogeneous data resources in support of clinically-relevant TBI methods; and 4) the similar design and delivery of computation methods that can act upon such architectures and translate bio-molecular discoveries into clinically actionable evidence, guidelines, or equivalent knowledge constructs. All of these conceptual foci can be aligned across a thematic spectrum from data discovery or management to the contextualization of said data in the form of information to the delivery of information in the form 


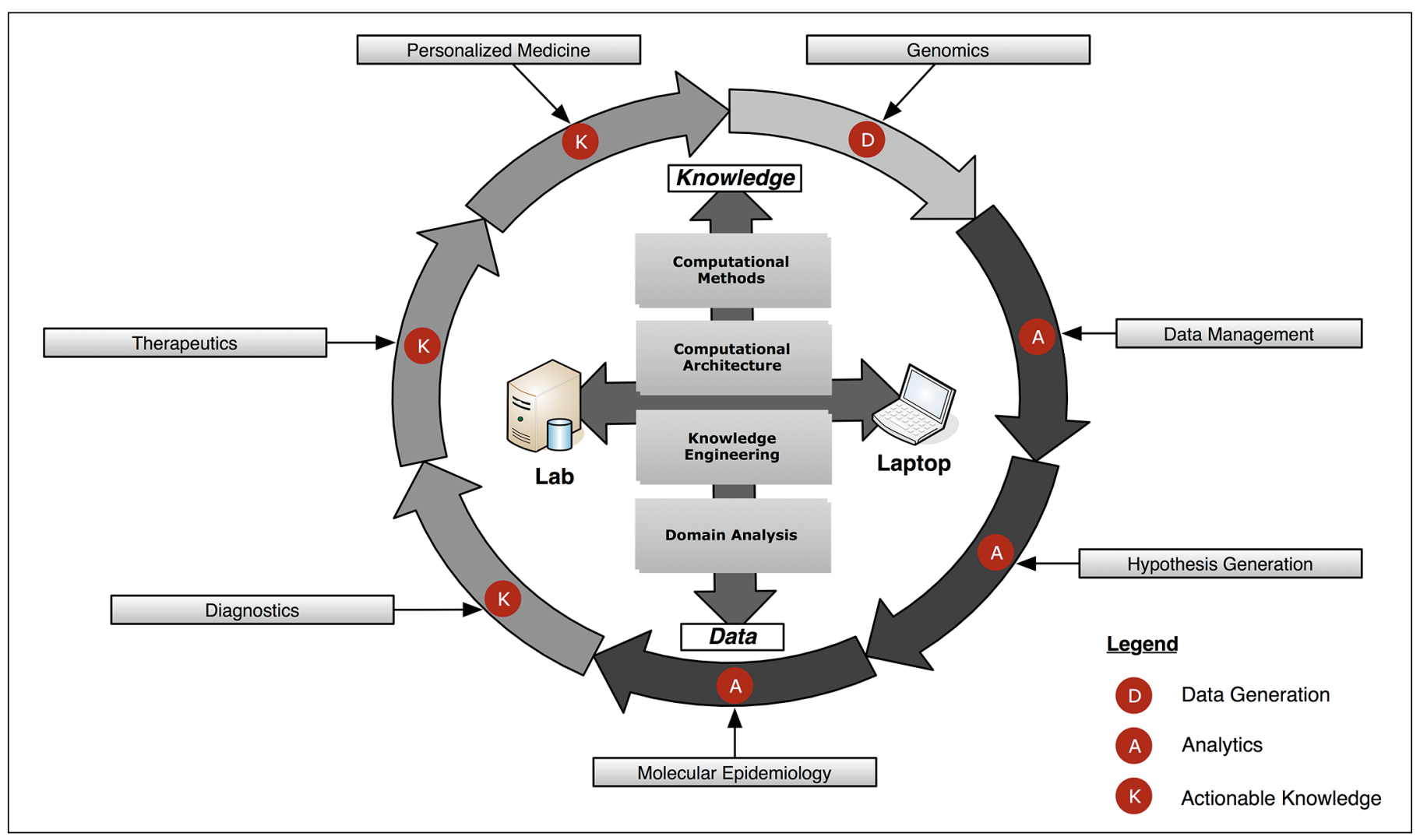

Fig. 1 Overview of organizing themes identified via analyses of selected literature corpus

of actionable knowledge, thus aligning with the central dogma of the broad field of Biomedical Informatics. In addition, these same conceptual foci can also be aligned across an application-oriented spectrum from lab to laptop, specifically connecting a range of activities beginning with bio-molecular discovery (in the lab) and ending with the in-silico facilitated or mediated application of ensuing scientific knowledge at the pointof-care or population health intervention (via the laptop as a metaphor for broader computational approaches).

- In a similar manner, the selected corpus of literature exhibited an alignment with seven methodological themes, corresponding to three major stages of knowledge generation and application, as follows: 1) in the first stage of knowledge generation and application, namely data generation, a primary focus of the literature was on the patient- or population-centric production of genomic data sets at varying levels of resolution; 2) in the second stage, namely the use of analytics methods, a primary focus on the literature was on data management (e.g., the collection, storage, and retrieval of integrative data sets), hypothesis generation (e.g., the automated and/or semi-automated creation of rationale questions to be explored in terms of genotype-to-phenotype relationships in targeted data sets), and molecular epidemiology (e.g., the observation of higher-order and population level patterns or motifs at the intersection of genotype and phenotype characteristic); and 3 ) in the third and final stage, namely, the delivery of actionable knowledge, the primary focus of the literature was upon the derivation of in silico methods or markers for some combination of diagnostics (e.g., disease risk profiling, diagnosis, or staging), therapeutics (e.g., the design and delivery of targeted therapeutic agents or interventions), and personalized medicine (e.g., the combined implementation and use of the aforementioned diagnostics and therapeutics approaches at a patient-specific level).
- Finally, and perhaps most importantly, all of the preceding foci and themes could be systematically aligned or organized into a virtuous cycle, via which their inputs and outputs (e.g., data, information, and knowledge) were both synergistic and self referential. This last finding is important in terms of recognizing the highly adaptive and systems level construct in which all such clinically relevant TBI theories and methods can and should be placed.

\section{Limitations}

Our approach to reviewing the current literature as a proxy for the state-of-the-art in clinically-relevant TBI research and development was targeted and involved a series of deliberate heuristic decision-points intended to control and manage the scope of such an assessment. As such, this report does not represent a systematic review of the domain 
and could omit any number of important findings or contributions not identified per the methods as described previously. Our analysis was also limited by the requirement for articles to include the term "translational bioinformatics" or "TBI." Furthermore, the thematic analyses presented in the Discussion were performed and subject to face-validity checking by the two authors of this article, and therefore represent a convenience sample of expertise and capabilities. In response to these potential limitations, we intend to expand and enhance this initial review in the form of a comprehensive and systematic literature review and corresponding stakeholder needs assessment in the near future. However, despite such limitations, we believe that this review provides a valuable and initial insight into the current state and practice of clinically relevant TBI research and development.

\section{Conclusions}

The field of TBI focuses upon the development and application of novel theories and methods capable of translating bio-molecular discoveries into actionable knowledge that can be used at the point-of-care or in population-level contexts in order to advance health and wellness. While significant and notable advances in foundational theories and methods relevant to TBI have been reported in the peer-reviewed literature, reports of implementation level efforts intended to apply such theories and methods to clinical decision making remain relatively sparse. In this review and in response to the aforementioned gaps in knowledge and practice, we have examined the current state-of-the-art in terms of such clinical implementation of TBI-generated knowledge, and in doing so, identified a set of common thematic elements that can frame open research questions for clinically relevant TBI activities. Ultimately, such work at the enumerated translational junctures will be critical in terms of demonstrating the value of TBI innovation in advancing the clinical and life sciences.

\section{References}

1. Butte AJ. Translational bioinformatics: coming of age. J Am Med Inform Assoc 2008;15(6):709-14.

2. Weston AD, Hood L. Systems Biology, Proteomics, and the Future of Health Care: Toward Predictive, Preventative, and Personalized Medicine. J Proteome Res 2004 March;3(2):179-96.

3. Altman RB. Chapter 2: Introduction to Translational Bioinformatics Collection. PLoS Comput Biol2012; 8(12): e1002796.

4. Kim JH. New horizons in translational bioinformatics: TBC 2013. BMC Med Genomics. 2014; 7(Supp 1): 11.

5. Capriotti E, Nehrt NL, Kann MG, Bromberg Y. Bioinformatics for personal genome interpretation. Brief Bioinform 2012;13(4):495-512.

6. Simon R, Roychowdhury S. Implementing personalized cancer genomics in clinical trials. Nat Rev Drug Discov 2013;12(5):358-69.

7. Payne PRO. Chapter 1:Biomedical Knowledge Integration. PLoS Comput Biol 2012;8(12):e1002826. doi:10.1371/journal.pcbi.1002826.

8. Chute CG, Ullman-Cullere M, Wood GM, Lin SM, He M, Pathak J. Some experiences and opportunities for big data in translational research. Genet Med 2013;15(10):802-9.

9. Chen J, Qian F, Yan W, Shen B. Translational biomedical informatics in the cloud: present and future. BioMed Res Int 2013;2013:658925. doi: 10.1155/2013/658925.

10. Shah NH, Cole T, Musen MA. Chapter 9: Analyses Using Disease Ontologies. PLoS Comput Biol 2012;8(12): e1002827. doi:10.1371/journal. pcbi.1002827.

11. Gonzalez MW, Kann MG. Chapter 4: Protein Interactions and Disease. PLoS Comput Biol 2012;8(12): e1002819.

12. Bebek G, Koyutürk M, Price DN, Chance MR. Network biology methods integrating biological data for translational science. Brief Bioinform 2012;13(4):446-59.

13. Sarkar IN. A vector space model approach to identify genetically related diseases. J Am Med Inform Assoc 2012;19(2):249-54.

14. Bhavnani SK, Abbas M, McMicken V, Oezguen N, Tupa J. iCircos: Visual Analytics for Translational Bioinformatics. IHI'12 Proceedings of the 2nd ACM International Health Informatics Symposium 2012;679-84

15. Lam TK, Spitz M, Schully SD, Khoury MJ. "Drivers" of translational cancer epidemiology in the 21st century: needs and opportunities. Cancer Epidemiol Biomarkers Prev 2013;22(2):181-8.

16. Deyati A, Younesi E, Hofmann-Apitius M, Novac $\mathrm{N}$. Challenges and opportunities for oncology biomarker discovery. Drug Discov Today 2013;18(1314):614-24.

17. Chen J, Zhang D, Yan W, Yang D, Shen B. Translational bioinformatics for diagnostic and prognostic prediction of prostate cancer in the next-generation sequencing era. BioMed Res Int 2013;2013:901578.

18. Lesko LJ. Drug Research and Translational Bioinformatics. Clin Pharmacol Ther 2012;91(6):960-2.

19. Butte AJ, Ito S. Translational Bioinformatics: Data-driven Drug Discovery and Development. Clin Pharmacol Ther 2012;91(6):949-52.

20. Hurle MR, Yang L, Xie Q, Rajpal DK, Sanseau P, Agarwal P. Computational drug repositioning: from data to therapeutics. Clin Pharmacol Ther 2013:93(4):335-41

21. LePendu P, Liu Y, Iyer S, Udell MR, Shah NH. Analyzing Patterns of Drug Use in Clinical Notes for Patient Safety. AMIA Jt Summits Transl Sci Proc 2012;2012:63-70.

22. Duke JD, Han X, Wang Z, Subhadarshini A, Karnik SD, Li X, et al. Literature Based Drug Interaction Prediction with Clinical Assessment Using Electronic Medical Records: Novel Myopathy Associated Drug Interactions. PLoS Comput Biol 2012;8(8): e1002614. doi:10.1371/journal. pcbi.1002614.

23. Johnson DE, Sudarsanam S, Bingham J, Srinivasan S. Translational Biology Approach to Identify Causative Factors for Rare Toxicities in Humans and Animals. Curr Drug Discov Technol 2012;9(1):77-80.

24. Azuaje F. Drug interaction networks: an introduction to translational and clinical applications. Cardiovasc Res 2013;97(4):631-41.

25. Dalpé G, Joly Y. Opportunities and challenges provided by cloud repositories for bioinformatics-enabled drug discovery. Drug Development Research. 2014;75(6):393-401.

26. Mirnezami R, Nicholson J, Darzi A. Preparing for Precision Medicine. N Engl J Med 2012;366(6):489-91.

27. Tarczy-Hornoch P, Amendola L, Aronson SJ, Garrawy L, Gray S, Grundmeier RW, et al. A survey of informatics approaches to whole-exome and whole-genome clinical reporting in the electronic health record. Genet Med 2013;15(10):824-32.

28. Suh KS, Sarojini S, Youssif M, Nalley K, Milinovikj N, Elloumi F, et al. Tissue banking, bioinformatics, and electronic medical records: the front-end requirements for personalized medicine. J Oncol 2013;2013:368751.

\section{Correspondence to:}

Philip R.0. Payne, PhD, FACMI

The Ohio State University

Department of Biomedical Informatics

250 Lincoln Tower

1800 Cannon Drive

Columbus, OH 43210, USA

Tel: + 16142924778

E-mail:philip.payne@osumc.edu 